Management international

International Management

Gestiòn Internacional

\title{
L'implication des enseignants : une des clés possible de la performance éducative
}

\author{
Teacher Involvement: One of the Keys to Educational \\ Performance
}

\section{La participación de los docentes: una de las claves del rendimiento educativo}

\section{Sandrine Fournier}

Volume 23, numéro 3, 2019

Le management public entre confiance et défiance

Public Management Between Trust and Distrust

Gestión pública entre la confianza y la desconfianza

URI : https://id.erudit.org/iderudit/1062208ar

DOI : https://doi.org/10.7202/1062208ar

Aller au sommaire du numéro

Éditeur(s)

HEC Montréal

Université Paris Dauphine

ISSN

1206-1697 (imprimé)

1918-9222 (numérique)

Découvrir la revue

Citer cet article

Fournier, S. (2019). L'implication des enseignants : une des clés possible de la performance éducative. Management international / International Management / Gestiòn Internacional, 23(3), 45-55. https://doi.org/10.7202/1062208ar

\section{Résumé de l'article}

Par le biais d'une étude menée dans les établissements publics locaux d'enseignement sur le territoire, nous nous intéressons à la résonance de l'implication des enseignants sur leur propre performance et celle des organisations publiques d'éducation. Nos résultats dévoilent ainsi plusieurs formes d'implication des enseignants avec des répercussions avérées sur leur performance et un prolongement de celle-ci sur la performance globale des établissements scolaires.
Tous droits réservés (C Management international / International Management / Gestión Internacional, 2019
Ce document est protégé par la loi sur le droit d'auteur. L'utilisation des services d’Érudit (y compris la reproduction) est assujettie à sa politique d'utilisation que vous pouvez consulter en ligne. 


\title{
L'implication des enseignants : une des clés possible de la performance éducative
}

Teacher Involvement:

One of the Keys to Educational Performance

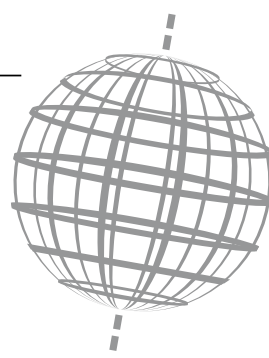

\section{La participación de los docentes: una de las claves del rendimiento educativo}

\author{
SANDRINE FOURNIER \\ Chercheuse indépendante rattachée à l'IRGO \\ Université de Bordeaux
}

\section{RÉSUMÉ}

Par le biais d'une étude menée dans les établissements publics locaux d'enseignement sur le territoire, nous nous intéressons à la résonance de l'implication des enseignants sur leur propre performance et celle des organisations publiques d'éducation. Nos résultats dévoilent ainsi plusieurs formes d'implication des enseignants avec des répercussions avérées sur leur performance et un prolongement de celle-ci sur la performance globale des établissements scolaires.

Mots clés : implication, performance, enseignants, établissements scolaires

\section{ABSTRACT}

Through a study carried out in local public educational establishments in France, we are interested in the impact of teachers' involvement on their own performance and the consequences on that of public educational organizations. Our results reveal several forms of teachers' involvement with proven consequences on their performance together with an impact on the overall performance of schools themselves.

Keywords : Involvement, performance, teachers, schools

\section{RESUMEN}

A través de un estudio realizado en instituciones educativas públicas locales en el área, nos interesa la resonancia de la participación de los docentes en su propio desempeño y el de las organizaciones de educación pública. Nuestros resultados revelan varias formas de participación de los maestros con un impacto comprobado en su desempeño y una extensión del mismo en el desempeño general de las escuelas.

Palabras clave : participación, rendimiento, profesores, escuelas

\section{Introduction}

$\mathrm{L}$ a mesure de la performance du système éducatif -définie par Lle nombre de jeunes titulaires d'un diplôme à la fin de leur scolarité- représente pour de nombreux pays une préoccupation majeure. La non obtention d'un diplôme engendre des corollaires économiques, sociales, financières directes et indirectes pour une nation. Dans le cas, de la France, le ministère de la défense relève en 2016, ${ }^{1}$ un pourcentage de $22,5 \%$ de jeunes fragiles au niveau de la lecture, soit une progression négative par rapport à l'année précédente. Sur les 760000 jeunes $^{2}$ évalués lors de la journée de défense et de citoyenneté (JDC), 5,1\% rencontrent des difficultés sévères. Malheureusement, ces chiffres montrent le nombre croissant d'élèves français susceptibles de ne pas maîtriser les compétences nécessaires pour s'insérer sur le marché du travail. Les chiffres internationaux publiés en 2016 par l'Organisme de Coopération et de Développement Economique (OCDE) intitulés (PISA) Programme International de Suivi des Acquis corroborent les résultats nationaux édités par la DEPP.
Sur les 72 pays évalués, ils démontrent pour la France une baisse globale des résultats scolaires pour les jeunes âgés de 15 ans et place ainsi le territoire au 26ème rang ${ }^{3}$. Ce phénomène est atypique et unique, sur l'ensemble des pays mesurés. En effet, la moyenne des élèves très performants est supérieure à ce qu'elle devrait être (18,4\% contre $15,3 \%$ pour la moyenne de l'OCDE), et à l'inverse, le nombre d'élèves peu performants est supérieur avec $14,8 \%$ contre $13 \%$ pour la moyenne de l'OCDE. ${ }^{4} \mathrm{La}$ France est le seul pays sur ceux évalués au niveau mondial à posséder cette discordance, alors que la formation est placée au centre des priorités du gouvernement français depuis plusieurs décennies. Partant de ces enjeux sociétaux, où de multiples outils et moyens ont été usités, nous nous préoccupons dans cette recherche de l'aspect humain et managérial afin d'identifier de potentielles pistes d'amélioration.

Nous étudions ainsi la problématique suivante : Comment l'implication des enseignants peut impacter la performance des établissements scolaires?

1. En 2015, sur 770000 jeunes, un pourcentage de $19,3 \%$ de jeunes fragiles au niveau de la lecture, dont $4.3 \%$ sont considérés comme illettrés et associés à une situation de handicap.

2. Ministère de la defense - DSN,MENESR- DEPP, note d'information $n^{\circ} 14$-mai 2017)

3. Mathématiques ( $\left.26^{\mathrm{eme}}\right)$, en compréhension de l'écrit ( 20 ème) et en sciences (26 ème).

4. Sans surprise, les pays asiatiques dominent le haut du classement avec à la première place Singapour pour les trois domaines évalués que sont les sciences, les mathématiques et la compréhension de l'écrit. Pour l'Europe, l'Estonie obtient le meilleur classement avec respectivement les 3 èmes, 6 ème et 9 ème places. Pour les Amériques, le Canada est en haut du podium avec les 7 ème, 2 ème et 12 ème places. Enfin, le bas du classement est détenu par l'Algérie. 
Dans cette optique, nous souhaitons enrichir l'analyse à travers deux questionnements sous-jacents.

En premier lieu, l'effet des interconnections (Meyer, Becker, Vandenberghe, 2004) entre les différentes formes d'implication sur la performance individuelle et la performance collective et en deuxième lieu, le lien entre ces performances humaines et la performance des établissements scolaires (Pirola-Merlo, Hartel, Mann et Hirst 2002).

Sur le plan conceptuel, cette recherche permet d'établir les effets de l'implication des enseignants sur leur performance et de déterminer celle des établissements scolaires -au sens du ministère- par la publication des résultats scolaires.

Sur le plan pratique, cette recherche a un double objectif. Le premier consiste à identifier les différentes formes d'implication des enseignants comme des facteurs susceptibles de déclencher une performance individuelle et une performance collective. Le second permet d'évaluer les relations et effets possibles entre la performance humaine et la performance des établissements publics locaux d'enseignement (EPLE) attendue par les parties prenantes comme gage de qualité.

Cette recherche s'appuie sur une démarche méthodologique quantitative de nature hypothético-déductive positiviste. Elle présente les résultats d'une étude menée sur l'ensemble du territoire y compris l'outre-mer dans les établissements publics locaux d'enseignement par le biais de la diffusion d'un questionnaire en ligne sur un échantillon potentiel de 7000 envois.

Pour apporter des éléments de réponses, cet article se compose en trois parties. La première propose le cadre conceptuel qui expose les notions d'implication et de performance des enseignants, pour poursuivre sur la performance publique à travers les indicateurs de performance des établissements scolaires. Dans une seconde partie, nous présentons la démarche méthodologique pour finalement, développer sur une troisième partie nos résultats et notre discussion avant de conclure sur les apports de la recherche.

\section{L'implication des enseignants}

Le processus d'implication est défini comme l'attachement psychologique et physique d'un individu envers sa tâche et son organisation. Cet attachement se traduirait par une forte croyance de l'individu dans les buts et les valeurs de l'organisation, par une volonté d'exercer des efforts significatifs au profit de celle-ci et par un fort désir d'en rester membre (Mowday, Porter, Steer, 1982). Notion plurielle, l'implication comprend plusieurs formes : de l'individuel, l'implication dans la carrière (Greenhaus, 1971), l'implication au travail ou à un emploi particulier (Kanungo, 1982), l'implication à la profession (Lodhal et Kejner 1965, Neveu 1996), l'implication à la tâche (Decotis et Summers 1987, Thevenet 2000), au collectif, l'implication de cohésion, l'implication au syndicat (Gordon, Philpot, Burt, Thomson et Spiller 1982), l'implication dans la culture de l'organisation et l'implication stratégique jusqu'aux formes mixtes (l'implication organisationnelle de Meyer et Allen 1984).
Dans son étude sur l'implication des enseignants, Fournier (2015) observe qu'un enseignant compétent et efficace en classe peut être absent de toute autre forme d'implication. Cela peut se manifester de différentes manières comme : ne pas travailler en équipe ou en réseau, ne pas participer à la vie et au projet de l'établissement, se tenir à l'écart des activités syndicales, ne pas ou peu s'investir dans la vie sociale, culturelle, politique et économique, qu'elle soit locale, régionale ou nationale. L'enseignant, tout comme son génome, possède son profil et son «matériel» implicatif particulier (Fournier, 2015). Les chefs d'établissement devront piloter des équipes avec des enseignants aux antipodes comportementaux, entre ceux qui s'impliquent à tous les niveaux, ou à l'inverse, ceux qui se tiennent à distance de tout. Les chefs d'établissement devront adopter des pratiques contrastées. Il n'existe pas de profil type d'enseignant impliqué, seules quelques tendances se dessinent en fonction du ressenti de chacun, de son implication au métier et/ou à l'organisation (Fournier, 2015). Les profils oscilleront entre des enseignants soucieux de collaborer en équipe, investis dans leur mission au sein de l'organisation et dans le projet d'établissement, ou à l'opposé des profils d'enseignants qui travaillent individuellement, sans se soucier de la politique de l'établissement et se désengagent de tout.

En sciences de l'éducation, Duchesne et Savoie-Zajac (2005) montrent que l'implication des enseignants présentent des facteurs d'engagements d'ordres privés tels qu'aimer les enfants, avoir le soutien des collègues ou de la direction. Les marques de reconnaissances du travail accompli et la possibilité d'actualiser ses connaissances incitent ces derniers à s'engager dans leur travail. En revanche, tout environnement opposé non favorable tels que le doute, les difficultés personnelles et les obstacles rencontrés dans la profession auront pour effet d'affaiblir, voire même d'anéantir, cette implication.

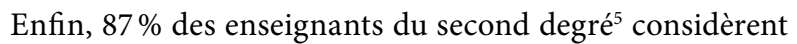
comme un gain le fait de pouvoir pratiquer une profession dans laquelle ils peuvent vivre leur passion, apprendre continuellement et vivre dans un contexte scolaire où sont présentes les ressources. Ils entretiennent un rapport impliqué à leur propre pratique, ils sont fiers d'exercer leur métier avec un réel désir pour la plupart de continuer d'exercer.

\section{La performance des enseignants}

Deux approches théoriques différentes issues des sciences de l'éducation expliquent la performance des enseignants. La première analyse l'influence des compétences des enseignants qui poursuivent leur apprentissage personnel par rapport aux collègues Cosnefroy (2004). Ces apprentissages engendrent une performance supérieure et apportent une satisfaction liée à la performance supplémentaire perçue. La deuxième approche détermine «l'effet enseignant» comme un facteur supplémentaire de performance par rapport aux facteurs périphériques déjà reconnus comme, des éléments déterminants à savoir : l'élève, le milieu familial et l'école (Cusset, 2011; Hattie, 2012; Gauthier, Bissonnette, Richard, 2013). Hattie (2012) ${ }^{6}$ a mis en avant le rôle primordial de l'enseignant, de son curriculum

5. Direction de l'Evaluation de la Prospective et de la Performance (2007) enquête menée auprès d'enseignants du secondaire

6. Enquête canadienne longitudinale sur de près de 15 ans réalisée sur plus de 240 millions d'élèves. 
et des méthodes d'enseignement utilisées sur la performance supplémentaire des élèves au-delà des dimensions familiales. Ces recherches reconnaissent une "valeur ajoutée» des compétences et méthodes usitées par les enseignants sur la performance des élèves.

Dans le cadre des sciences de gestion, les agents publics de première ligne sont censés poursuivre de nouveaux objectifs, de plus en plus axés sur la performance, même si les attentes classiques «légalité, égalité, fraternité» sont toujours présentes, (Pollitt et Bouckaert, 2004; Emery et Giauque, 2005). Les auteurs Emery, Wyser, Martin, Sanchez (2008) précisent que «la performance doit être clairement appliquée à tous les échelons de la hiérarchie, un processus difficile étant donné que la NGP (Nouvelle Gestion Publique) a fait apparaître de nouvelles valeurs susceptibles d'entrer en conflit avec les valeurs traditionnelles du secteur public», ce qui suscite des injonctions contradictoires.

Les publications qui analysent la performance par le personnel soulignent toutes, le fait, que la performance dans le secteur public doit obligatoirement être considérée comme une notion multidimensionnelle, bien plus complexe que les indicateurs quantitatifs axés sur les résultats essentiellement utilisés dans les accords de rendement s'inspirant d'une perspective managériale des services publics (Pollitt 2000). Malgré la «managérialisation " progressive, le secteur public reste public, non axé sur le profit et soumis à un cadre juridique spécifique qui garantit la légalité et l'équité pour les citoyens, créant ainsi un environnement hybride (Jorgensen, 1999).

En outre, l'utilisation de pratiques plus humanisées, associées au partage de l'information, le développement des compétences (feedback reçu sur la performance exécutée jusqu’à la reconnaissance non monétaire) sont favorables à une meilleure performance (Wood et Albanese, 1995). Ainsi, la performance au travail serait un élément central de l'estime de soi et l'implication serait appréhendée comme le résultat de l'évaluation personnelle de l'adéquation entre sa performance individuelle et les capacités requises (Vroom, 1964); (Aronson et Carlsmith, 1962). Il est nécessaire d'apporter une attention particulière à la ressource humaine en place pour espérer agir sur un niveau de performance (Rhoades, Eisenberger, Armeli, 2001). Cette performance individuelle (Motowildo, 2003) se rapporte à des comportements que l'organisation met en avant et qu'elle espère de ses salariés. C'est pourquoi, cette définition sous-tend que l'individu peut être très performant sur certaines actions et moins sur d'autres, mais également que sa performance peut changer au cours du temps pour des motifs tels que le stress. Ainsi, Williams et Anderson (1991) ont intégré les effets du stress dans leur étude sur l'atteindre de la performance individuelle et l'analyse des comportements attendus. D'autres recherches sur la performance dans la société mouvante, notamment celle de Pulakos (2000), définit la capacité des individus à s'adapter aux nouvelles conditions et exigences du travail telles que les situations de crise et la gestion du stress.

\section{La performance publique}

Les notions de résultats et de performance émergent dans les établissements publics (Emery et Giauque, 2005); (Carassus, Favoreu, Gardey, Marin, 2012; Mazouz, Garzon, Picard, 2012) avec une évolution du concept au gré des réformes administratives (Bartoli et Chomienne, 2007), (Chomienne, 2001), (Guenoun, 2009). La culture de la performance en France se trouve au centre de la Loi Organique relative aux Lois de Finances (LOLF 2001) avec l'instauration des indicateurs de productivité et de performance. Le développement de la culture du résultat a conduit à la mise en place ambitieuse de l'évaluation des politiques publiques (Basle, 2009a) et à l'introduction progressive de la nouvelle gouvernance publique. Deux logiques concomitantes se côtoient, la minimisation des coûts (logique budgétaire) et la maximisation des services publics (logique sociale). L'article 51-5 de la LOLF prévoit ainsi d'étendre un projet annuel de performance pour chaque programme contenant «la présentation des actions, des coûts associés, des objectifs poursuivis, des résultats obtenus et attendus pour l'année à venir, mesurés au moyen d'indicateurs précis dont le choix est justifié». L'effet de la LOLF est la réalisation d'un management public comme conséquence d'une structure budgétaire (Rouban, 2008). Sous l'action des politiques publiques, Bezes et Demazière, (2011) décrivent le NMP comme « un puzzle doctrinal constitué de strates variées d'éléments de la théorie économique, de préceptes issus des sciences de gestion et de prescriptions inspirées des réformes antérieures de l'administration».

Gibert (2008), définit l'organisation performante selon une approche focalisée sur l'efficience, c'est-à-dire l'atteinte des objectifs avec une consommation de moyens relativement restreinte. L'utilisation minimale des ressources et des moyens permettra d'alimenter l'efficience de l'organisation. Pour le ministère du budget, il s'agit de la capacité à atteindre des objectifs préalablement fixés, exprimés en termes d'efficacité socio-économique, de qualité de service ou d'efficience de la gestion. La performance publique peut donc être intégrée comme la faculté des organisations publiques à mettre en œuvre les politiques publiques et à rendre compte de leurs résultats (Waintrop et Chol, 2003).

La démarche d'analyse et d'évaluation de l'ensemble des politiques publiques ${ }^{7}$ se poursuit, par la mise en place de démarches de contrôle de la performance publique, ainsi 59 analyses ont été enclenchées depuis $2012^{8}$. Nous signalons en adéquation à la définition de Gibert (2008) sur «l'atteinte des objectifs avec une consommation de moyens relativement restreinte», deux évaluations en correspondance à notre thématique. L'évaluation de la performance publique dans le cadre de l'éducation nationale s'effectue autour de l'évaluation de la gestion des heures d'enseignement au regard de la carte des formations $s^{9}$ et le développement et l'optimisation de ressources financières et humaines ${ }^{10}$. Notre recherche sur le levier de l'implication des enseignants comme déclencheur de performance rentre pleinement dans le cadre de ces deux évaluations sur l'optimisation à la fois financière et humaine du «capital humain» en place dans les EPLE.

7. L'évaluation de l'école publique éditée en février 2015 et accessible sur le site de modernisation de l'école.

8. http://www.modernisation.gouv.fr/laction-publique-se-transforme/en-evaluant-ses-politiques-publiques/toutes-les-evaluations-de-politiques-publiques

9. Inspection Générale de l'Administration de l'Education Nationale et de la Recherche (2014) nº 2014-035 de juin 2014.

10. Rapport octobre 2015 «Evaluation du partenariat de l'Education Nationale et de l'Enseignement Supérieur avec le monde économique pour l'insertion professionnelle des jeunes» 


\section{La performance des établissements scolaires}

Les acteurs du système éducatif comme les recteurs ou les chefs d'établissement sont appelés avec la mise en application de la LOLF (2006) à performer sous forme de contrat d'objectifs ${ }^{11}$. L'évaluation de ces objectifs s'effectue au moyen d'indicateurs de résultats qui seront comparés avec ceux fixés par la représentation nationale et académique.

Les principaux indicateurs de mesure de performance des établissements scolaires ont évolué. Au départ en 1991, il existait seulement trois critères : le taux de réussite au baccalauréat, le taux d'accès de seconde et de première au baccalauréat (niveau supérieur) et la proportion de bacheliers parmi les sortants.

Depuis, les indicateurs évoluent vers des analyses plus poussées et pertinentes telles que :

- des indicateurs sur la destination des élèves à leur sortie du lycée,

- des indicateurs relatifs à la population prise en charge (sexe, Catégorie Socio-professionnelle des parents, proportion de redoublants, origines scolaires...),

- des indicateurs de ressources et de moyens (moyens horaires, effectifs, enseignants),

- des indicateurs de fonctionnement et d'environnement (proportion d'heures d'enseignements, effectif élèves par classe, offres de formations proposées, services vie scolaire, contacts avec le monde économique...).

Nous proposons en annexe 1 un tableau de synthèse construit à partir des données du Ministère de l'Education Nationale pour l'académie de Bordeaux-Poitiers (2016). Il illustre à partir des indicateurs de mesure de la performance un positionnement des EPLE.

Les établissements sont classés en cinq catégories en référence à la notion de "valeur ajoutée", c'est-à-dire la capacité des établissements à faire progresser les élèves en fonction de l'âge, de l'origine sociale et le niveau scolaire des jeunes et non pas, au regard du taux de réussite au baccalauréat.

- Les lycées performants obtiennent des résultats plus élevés (valeur ajoutée positive et significative) que les attentes du Ministère en termes de réussite au Baccalauréat et d'accès des élèves de la seconde au Baccalauréat.

- Les lycées accompagnateurs pour les établissements privilégiant un suivi de tous les lycéens de la seconde jusqu'au Baccalauréat.

- Les lycées sélectifs avec un écrémage de la seconde à la terminale afin de garantir un taux de succès supérieur à la moyenne.

- Les lycées neutres pour la majorité des lycées dont les indicateurs approchent des valeurs prévues avec une valeur ajoutée faible sur les taux de succès ou d'accès de la seconde.
- Les lycées « en-deçà des attentes» lorsque les deux indicateurs sont inférieurs aux prévisions pour un lycée à population et structure de formation égale.

Nous constatons que seuls 9,30\% des établissements éducatifs sont classés comme performants soit 29 sur un total de 312 alors que 104 soit $33,33 \%$ sont «en deçà des attentes ». Une lecture plus poussée réservée aux spécialistes et responsables académiques permet d'analyser établissement par établissement l'utilisation des moyens alloués, les stratégies d'orientations éducatives. Ces résultats combinés aux critères suivants : le nombre d'élèves, la variété des filières, les disciplines proposées, la géographie sociale fournissent des éléments de réflexion, d'analyse pour aider les chefs d'établissements à améliorer l'efficacité de leurs actions et gérer au mieux la ressource humaine. Chaque année, le ministère de l'Éducation Nationale le répète : les indicateurs de résultats des lycées publiés n'ont pas vocation à constituer un "classement» mais ils doivent offrir aux équipes pédagogiques, aux parents et aux élèves "une image de la réalité complexe et relative que constituent les résultats d'un établissement ». Or, l'évolution des diverses mesures et indicateurs suggèrent qu'il y a un résultat à atteindre et suppose que toute action publique ne peut être appréciée que par référence à une conformité aux objectifs (Chappoz et Pupion, 2013). Les attentes de résultats et de performance pourraient provoquer des tensions et des pressions supplémentaires auprès des acteurs du système éducatif (Fournier, 2015). L'auteure précise qu'une approche «consumériste» s'impose au sein du système éducatif français. Certes, «aucun système de mesure de la performance n'est parfait, il doit donner lieu à des comparaisons entre les structures, faciliter leur pilotage et s'adapter aux besoins spécifiques de chacune » Corbel (2015). Cependant, cette logique est susceptible de provoquer un glissement des inscriptions vers les établissements performants sur la logique de "loi du marché» et de la concurrence avec des conséquences pour les établissements «en deçà de leur possibilité». D’autres retentissements pourraient en découler tels que la non attractivité des recrutements d'enseignants dans des zones dites "difficiles». La publication annuelle des résultats aux examens pourraient remettre en question l'équité du système éducatif et provoquer des discordances de notoriété des établissements à plus ou moins court terme et moyen terme.

\section{Les effets de l'implication sur la performance}

L'adhésion d'une personne aux valeurs et au projet de l'organisation est nécessaire, elle préfigure un investissement au travail et une volonté d'agir (Thevenet, 1992). Confier des tâches variées aux salariés, les inciter à utiliser de multiples compétences, à les doter d'autonomie, à donner du sens et de la visibilité à l'ensemble des tâches qu'ils exécutent sont considérés comme un moyen efficace d'influer sur leurs attitudes et comportements au travail (Manville, 2014).

11. Le «contrat d'objectifs» est la mise en ouvre d'une circulaire d'application de la loi d'orientation et de programme pour l'avenir de l'école et de la loi de programmation pour la cohésion sociale. Pour chaque établissement, doit être établi un projet de contrat d'objectifs en cohérence avec le projet d'établissement, et sur la base des orientations fixées au niveau national et académique. Il définit des objectifs à atteindre à une échéance pluriannuelle (de 3 à 5 ans) sous forme d'un programme d'actions. Les indicateurs permettant d'apprécier la réalisation de ces objectifs sont mentionnés dans le contrat. Ce projet de contrat est élaboré dans le cadre d'un dialogue avec l'autorité académique puis après approbation, il est signé par le recteur ou son représentant et le chef d'établissement. 
Le niveau d'implication affective (Allen et Meyer, 1984), est celui qui apparaît comme le plus fortement prédictif de comportements bénéfiques aux organisations sur la performance au travail ou la fidélité (Mathieu et Zajac, 1990; Meyer et al., 2002; Morrow, 2011). Les personnes impliquées travaillent plus et réussissent mieux que celles ayant une faible implication. Cette attitude permet de favoriser des effets plus productifs au travail pouvant créer par conséquent de la valeur (Walumbwa et al. 2008).

D'autres pratiques de GRH (Pfeffer et Veiga, 1999; Ferris et al. 1999) sont considérées comme cruciales à l'avantage concurrentiel et démontrent une relation positive entre la gestion stratégique de l'humain et l'impact sur la performance (Delery et Doty, 1996; Huselid, 1995; Mac Duffie, 1995; Pfeffer et Veiga, 1999). Arcand, Bayad, Fabi (2002) précisent que l'attention apportée aux ressources humaines peut être associée de façon significative à l'amélioration des indicateurs de performance concernant la communication, l'organisation du travail, l'évaluation du rendement et la rémunération. Les compétences des salariés, la valeur de leur engagement, leur productivité seront à la base de la réussite de l'organisation à laquelle ils appartiennent (Charreaux, 1998).

Plusieurs tendances se distinguent au niveau mondial sur les effets de l'implication des agents sur la performance publique (OCDE, 2005). La question du lien entre les résultats des évaluations de la performance et les récompenses accordées au personnel se poserait actuellement dans bon nombre de pays de l'OCDE (2017), avec de nombreuses réformes en cours. ${ }^{12}$ Certains pays privilégient une politique de Rémunération Liée à la Performance (RLP) à grande échelle dans leur fonction publique, par l'évaluation de la capacité des cadres dirigeants à mener des réformes et la responsabilisation des hauts fonctionnaires.

Pour exemple, deux mesures sont utilisées en Corée avec la Direction Par Objectifs (DPA) pour les cadres et la notation de la performance pour les personnels de rangs intermédiaires et subalternes au moyen d'une évaluation à 360 degrés.

\section{Modèle et hypothèses}

Pour l'élaboration de notre modèle présenté en annexe 3, nous examinons les relations entre les différentes formes d'implication sur la la performance individuelle et la performance collective des enseignants ainsi que les relations entre ces performances sur celle des établissements scolaires. Nous supposons les effets de l'implication sur la performance humaine avec des impacts de ces dites performances sur celle des établissements scolaires.

Ces arguments conduisent à formuler les hypothèses selon lesquelles :

Hypothèses 1a et $1 \mathrm{~b}$ : L'implication influe sur la performance individuelle et sur la performance collective des enseignants.

Hypothèses $2 \mathrm{a}$ et $2 \mathrm{~b}$ : Les différentes dimensions collectives et individuelles de la performance (l'auto-efficacité ou sa capacité individuelle à atteindre les objectifs visés) agissent sur la performance des élèves et de l'organisation.

\section{Méthode de recherche}

\section{Participants et PRocédure}

Cette étude a été conduite dans les établissements publics locaux d'enseignement au niveau national y compris l'outremer auprès d'une population d'enseignants du secondaire. Nous avons contacté des personnels titulaires, non titulaires travaillant dans différentes catégories d'établissements (lycées généraux, lycées techniques et professionnels) implantés en zones rurales ou urbaines. L'enquête s'est déroulée au moyen d'un questionnaire de type Likert à 7 niveaux (de pas du tout en accord à très fortement en accord) par une diffusion par voie électronique auprès d'un échantillon de 7000 enseignants. Nous avons obtenu peu de retour et seul 80 questionnaires ont été exploitables. La structure de l'échantillon est la suivante : $60 \%$ d'hommes avec un âge moyen de 40 ans et une ancienneté moyenne de 15 ans, et de $40 \%$ de femmes âgées en moyenne de 38 ans avec une ancienneté moyenne de 10 ans. La répartition des lieux d'enseignement des enseignants s'articule comme suit : $25 \%$ enseignent en zone rurale, $65 \%$ en zone urbaine et $10 \%$ en ZEP (Zone d'Education Prioritaire). Nous avons choisi d'associer une observation active longitudinale dans un lycée professionnel de Gironde durant quatre années scolaires composé d'un effectif de 88 enseignants. L'ensemble des données recueillies a été traité au moyen du logiciel statistique SPSS.

\section{ECHELLES DE MESURE MOBILISÉES}

Dans un premier temps, nous avons procédé à la purification de la base de données de la première collecte par une analyse factorielle et par le calcul de l'alpha de Cronbach de chaque dimension de l'échelle. Nous avons obtenu des échelles épurées. Seules, les variables dont la signification est supérieure à 0,7 ont été conservée afin d'obtenir des énoncés valides et fiables sous un plan empirique.

Pour l'évaluation de la notion d'implication multiple, nous avons, après l'épuration, retenu un total de 41 items répartis en 3 dimensions.

- Pour la première dimension sur l'implication psychologique (Lodalh 1965, Lodalh et Kejner 1965, Kanungo 1982 puis Neveu 1996), nous avons un nombre de 10 items,

- Pour la deuxième dimension sur l'implication affective, normative et calculée (Allen et Meyer, 1990), nous avons un nombre de 14 items,

- Pour la troisième dimension sur implication professionnelle (Goodell, 1969), nous avons retenu deux sous dimensions : une composée de 9 items pour évaluer l'effet des conditions de travail, et la deuxième composée de 8 items pour évaluer l'engagement dans l'organisation.

Pour l'évaluation de la notion de performance multiple, nous avons, après épuration, retenu un total de 16 items répartis en trois dimensions.

- Pour la première dimension sur la dynamique collective de la performance (Borman, Buck and al. 2001; Coleman, Borman 2000), nous avons un nombre de 4 items,

12. Dix-neuf pays de l’OCDE se sont dotés d'un mécanisme spécifique de gestion de la performance des hauts fonctionnaires, tandis que dans 9 autres pays, les hauts fonctionnaires sont soumis au même régime de performance que l'ensemble des fonctionnaires. 
- Pour la deuxième dimension sur la performance individuelle ou auto efficacité (Williams et Anderson 1991), nous avons un nombre de 7 items,

- Pour la troisième dimension sur la performance et l'association stress-performance (Williams et Anderson 1991), nous avons un nombre de 5 items. Nous présentons les caractéristiques de l'ensemble des items dans l'annexe 2.

Dans un deuxième temps, nous avons exploité les principaux résultats de l'analyse quantitative à partir d'une analyse univariée et bivariée des données récoltées. Les traitements bivariés ont pour objet de mettre en évidence les relations éventuelles qui existent entre deux variables analysées simultanément. Pour nos échelles de mesure, nous choisissons d'utiliser les indicateurs de corrélations du test de Spearman (rhô) pour vérifier la corrélation entre les variables.

\section{Résultats}

Les résultats des croisements significatifs (annexe 4) indiquent une quantité importante de corrélations positives de l'implication sur la performance individuelle et un nombre inférieur de corrélations sur la performance collective.

Nous relevons, un nombre restreint de relations entre la performance individuelle et collective sur la performance des établissements scolaires. En outre, à l'issue de l'analyse des croisements, nos résultats révèlent des relations directes de l'implication des enseignants à la fois sur la performance des élèves et, à la fois sur la performance des établissements scolaires comme en réfère notre modèle 2 (annexe 5).

\section{L'implication sur la performance individuelle}

L'analyse des résultats démontrent un nombre important de relations entre l'implication (psychologique, affective, calculée, normative et professionnelle) sur la performance individuelle des enseignants.

\section{L'IMPLICATION PSYCHOLOGIQUE}

Les enseignants sont impliqués psychologiquement à leur métier $(0,268)$, cet attachement associé à la qualité de vie (articulation du calendrier scolaire) $(0,311)$ leur permet, non seulement de résister en partie aux pressions et au stress $(0,171)$, mais, également d'être attentif aux performances de leurs élèves.

\section{L'implication affective, calculée et normative}

L'implication affective des enseignants est liée à une certaine performance individuelle par l'attention apportée à la réussite aux examens des élèves $(0,439)$. Le rythme de vie qu'offre cette profession leur permet de récupérer -éviter les effets du stresspour mieux performer $(0,276)$ et consacrer du temps au suivi des jeunes $(0,499)$. L'implication (calculée) des enseignants dans leur relation à leur organisation $(0,252)$, leurs années d'expérience d'enseignement $(0,212)$ associées, à leur organisation personnelle $(0,317)$ sont des éléments opportuns à la performance individuelle. Le devoir moral, l'attachement à la profession (implication normative) sont des facteurs favorables à la performance par un intérêt porté au suivi des élèves $(0,285)$.
Ainsi, l'équilibre de vie entre temps de travail et temps personnel favorise la relation de maintien de la performance $(0,205)$. En outre, nous relevons que l'implication affective $(-0,406)$ et calculée $(-0,210)$ des enseignants agit négativement sur leur auto efficacité vis à vis des élèves.

\section{L'IMPLICATION PROFESSIONNELLE}

Nous remarquons trois formes d'implication professionnelle en relation avec la performance individuelle : l'implication envers les élèves, l'implication envers les collègues et l'implication au travail.

Les enseignants prennent du plaisir dans la pratique de leur métier, notamment par la transmission de leurs connaissances vécues par eux, comme une source d'enrichissement personnel $(0,208)$, ils consacrent du temps au suivi des élèves $(0,349)$ et à leur réussite. Le travail tient une place notable dans la vie des enseignants $(0,207)$. Leur implication professionnelle individuelle au travail $(0,226)$ favorise leur performance individuelle. Nous relevons que l'implication professionnelle envers les collègues c'est-à-dire collaborer en équipe pédagogique active l'attention apporté à la performance des élèves $(0,530)$, mais pas à titre individuel $(-0,292)$. Nous notons que l'implication professionnelle supplémentaire des enseignants vis-à-vis des élèves agit négativement $(-0,229)$ à celle des élèves.

L'analyse globale des relations entre les différentes formes d'implications sur la performance individuelle, renforce l'ensemble des théories issues des sciences de l'éducation sur les notions de compétences (Cosnefroy, 2004), et «d'effet enseignant» (Cusset, 2011; Hattie, 2012) avec celles en sciences de gestion sur les effets de l'implication sur la performance individuelle (Vroom, 1964; Aronson et Carlsmith, 1962) de la capacité des individus à s'adapter aux exigences du travail et à la gestion du stress Pulakos (2000). Nous confirmons l'attachement et une forte croyance dans les buts et les valeurs de l'organisation, par une volonté d'exercer des efforts significatifs au profit de celle-ci (Mowday, Porter, Steer, 1982). Notre recherche confirme tout comme Walumbwa et al. (2008) que les personnes impliquées travaillent plus et réussissent mieux que ceux ayant une faible implication, que cette attitude permet de favoriser des effets plus productifs au travail pouvant créer par conséquent de la valeur.

\section{L'IMPLICATION SUR LA PERFORMANCE COLLECTIVE}

Nous relevons quelques relations entre l'implication affective, normative et professionnelle des enseignants sur la performance collective. L'implication affective $(0,186)$ est liée à la performance supplémentaire induite sous l'effet du groupe. Elle sera décuplée lors du travail d'équipe avec une volonté d'implication supérieure véhiculée par la collaboration et le collectif. La collaboration pédagogique active $(0,442)$ est primordiale pour la qualité du travail rendu par les enseignants dans les EPLE. Une bonne ambiance de travail $(0,310)$ favorise l'attachement au métier et à l'établissement et suscitent des phénomènes de synergie, de mutualisation déclencheurs d'une performance collective. Nous confirmons les effets de synergie, de cohésion, de collaborations (Gordon, Philpot, Burt, Thomson et Spiller, 1982) qui seront activés par l'implication professionnelle groupale. 


\section{LIEN ENTRE LA PERFORMANCE DES ENSEIGNANTS ET LA PERFORMANCE DES ÉTABLISSEMENTS SCOLAIRES}

Nous remarquons l'assujettissement de la performance individuelle sur la réussite des élèves $(0,229)$, grâce à une politique de suivi des classes d'une année sur l'autre. Dans cette logique de pratiques managériales avérées et par la pratique de la direction par objectifs sur les équipes pédagogiques $(0,229)$ les chefs d'établissement favorisent la performance groupale et directement la performance des établissements scolaires $(0,342)$.

Les enseignants impliqués sont un capital pour l'Education Nationale et particulièrement pour la structure dans laquelle ils travaillent. Les effets de leur implication multiple imputeront directement la performance individuelle et dans une moindre mesure la performance collective. Ce chaînon de corrélations influence la performance globale des établissements scolaires sous l'effet de pratiques managériales (suivi des classes, direction par objectifs) et principalement sous l'impulsion de la performance individuelle. Nous confirmons à travers nos résultats, que la performance au travail serait un élément central de l'estime de soi et l'implication serait appréhendée comme le résultat de l'évaluation personnelle de l'adéquation entre sa performance individuelle et les capacités requises (Vroom, 1964); (Aronson et Carlsmith, 1962).

Nous constatons que la performance individuelle des enseignants est liée à plusieurs éléments tels que :

L'attention apportée au suivi des résultats de leurs élèves,

- à une bonne compréhension de l'ensemble du fonctionnement organisationnel de l'établissement,

- à leurs divers rôles et missions dans une équipe,

- à la capacité de faire le lien entre leur rôle personnel et les objectifs organisationnels (projet d'établissement),

- et aux contrats d'objectifs académiques et nationaux.

Notre recherche montre un lien induit de la performance individuelle et groupale sur la performance des établissements, alors que les enseignants n'ont pas toujours cette vision. Pour eux, leur première mission est d'enseigner, de transmettre un savoir. Ils occultent l'évaluation de la performance telle quelle est notifiée dans la LOLF : nous irons même jusqu'à signaler une méconnaissance de son contenu et de ses aboutissants. Or, nos résultats prouvent que par leurs faits, leurs actes et leurs actions, les enseignants œuvrent pour l'amélioration de la performance publique.

\section{LES EFFETS DE L'impliCATION SUR LA PERFORMANCE DES ÉTABLISSEMENTS}

Nous constatons une relation commune directe à toutes les formes d'implication des enseignants sur le suivi des résultats des élèves c'est-à-dire : à la réussite aux examens et à la performance des établissements scolaires. L'implication psychologique, l'implication affective, l'implication calculée, l'implication normative, l'implication professionnelle envers les élèves, l'implication professionnelle envers les collègues, l'implication professionnelle au travail impactent toutes la performance des EPLE. Notre modèle montre que l'implication des enseignants dans leur mission, dans leur métier favorise directement la performance des élèves, même si, lors de nos observations, nous observons que la prise de conscience de cet impact n'est pas perçue ainsi par eux.

\section{Discussion}

A l'issue de cette présentation des résultats, nous mettons en avant la portée des leviers de gestion de la ressource humaine sur l'implication des enseignants dans le secteur public comme une source de leur performance avec des conséquences induites sur la performance des établissements scolaires. Nous rejoignons Rhoades et al. (2001) sur la nécessité d'apporter une attention particulière à la ressource humaine en place pour espérer agir sur le niveau de performance. Ainsi, les chefs d'établissement jouent un rôle primordial. Nous appuyons leur rôle de manager comme soutien à des attitudes et comportements favorables à l'implication et à la performance. Plus les chefs d'établissement donneront des conditions de travail favorables, telles qu'un emploi du temps souhaité, suivre les classes, une ambiance de travail adéquate, le choix des équipes pédagogiques, plus ils seront impliqués et performants (Fournier 2017). Wood et Albanese (1995) précisent que dans le domaine public, le partage de l'information, le développement des compétences, en passant par le feedback reçu agissent sur la performance.

Les résultats de notre recherche se déclinent en cinq principaux apports.

Le premier concerne l'impact notable de l'implication des enseignants sur la performance individuelle. Cette étude met en exergue la nécessité de reconnaître les valeurs du personnel dans le secteur public.

Le deuxième est de démontrer l'influence de l'implication des enseignants sur la performance collective. La dynamique de groupe, la collaboration et la synergie qui en découle sont des éléments favorables au maintien de l'implication et à la performance collective. Nous confirmons la relation positive entre une gestion stratégique des enseignants par les chefs d'établissements et un impact sur la performance comme un avantage concurrentiel indéniable (Delery et Doty, 1996; Huselid, 1995; Mac Duffie, 1995; Pfeffer et Veiga, 1999).

Le troisième est de mettre en avant la performance individuelle et collective des enseignants comme des gages de la performance des établissements scolaires en réponse aux indicateurs de performance attendus au niveau nationale et académique.

Le quatrième apport est de révéler l'impact direct de l'implication multiple des enseignants comme une des clés de la réussite de la performance des établissements publics locaux d'enseignements.

Enfin, le cinquième réside dans le développement de ce sujet sous un angle des sciences de gestion et ainsi montrer l'avantage concurrentiel des pratiques RH sur la contribution de création de valeurs par l'amélioration du rendement (Arcimoles et Trébucq, 2002).

Le maintien de relations positives au sein des EPLE sont cruciales et engendrent des conséquences positives à la fois sur l'implication et sur la performance humaine (Pfeffer et Veiga 1999, Ferris et al. 1999) avec des corollaires sur la performance induite dans les établissements scolaires.

\section{Conclusion}

Cette recherche a permis de positionner le rôle central de l'implication multiple des enseignants dans le contexte d'une amélioration de la performance du système éducatif français, 
sous condition de la mise en place de pratiques de gestion des ressources humaines appropriées par les chefs d'établissement. L'ensemble de ces implications forment des agrégats favorables à la logique de prise en compte de la dimension performance chères au NPM. Paradoxalement, la performance globale des établissements mesurée à travers les indicateurs de mesure de la performance n'est pas encore ressentie et perçue sous cet angle par les enseignants, alors qu' ils agissent directement sur celle-ci par leur implication multiple. Notre étude enrichit les connaissances actuelles sur l'effet des implications multiples comme des variables intermédiaires à la performance des enseignants et à celle des établissements scolaires. Enfin, nous soutenons que des pratiques centrées sur l'humain et spécifiques à la gestion des enseignants peuvent activer et favoriser l'amélioration de la performance du système éducatif.

En définitive, cette recherche se veut être une première étape dans l'exploration des effets de l'implication des enseignants sur la performance induite des établissements scolaires. En outre, la partie exploratoire mériterait d'être renforcée sous deux angles, la première par une étude d'une plus grande ampleur en tenant compte des perspectives territoriales différenciées et la seconde complémentaire vers une analyse des pratiques managériales menées par les chefs d'établissement. Dans cette perspective, plusieurs questions demeurent et émerguent à l'issue de ce travail comme, l'analyse des comportements au travail des agents public avec des transferts possibles vers d'autres organisations publiques. Comment chaque partie prenante s'engagent dans un projet de performance? Quels sont les effets perçus et rééls au niveau des personnels avec comme corrolaire la préservation d'un équilibre social et du bien être des agents. Nous continuons nos investigations sur la base d'un capital humain indispensable et vital aux organisations. Il est riche de sens, porteur de l'équilibre futur pour faire face aux mouvances extérieures telles que l'appropriation des nouvelles technologies, la mise en place de pratiques différenciées, les restructurations et les restrictions budgétaire à venir dans le domaine public.

De cette recherche découle la problématique de la légitimité des pratiques managériales attentives aux changements et conséquences sous l'action des personnels. La poursuivre des recherches dans le domaine de la gestion des ressources humaines sont primordiales, par l'observation et la mise en exergue des connaissances comme un moyen de traçabilité des pratiques à conduire. Les agents sont à la fois les piliers, les fondateurs, de l'histoire vivante des entités publiques et privés. Dans notre cas, la question reste ouverte qu'adviendrait-il au niveau organisationnel, si les phénoménes de désimplication ou d'épuisement professionnel se développaient?

\section{Bibliographie}

ArCAnD, Michel; BAyAD, Mohamed; FABI, Bruno (2002). «L'effet des pratiques de gestion des ressources humaines sur l'efficacité organisationnelle des coopératives financières canadiennes", Annals of Public and Cooperative Economics, Vol. 73, $\mathrm{n}^{\circ} 2$, p. 215-240.

Aronson, Elliot; Carlsmith, J.Merrill (1962). «Performance expectancy as a determinant of actual performance», Journal of Abnormal and Social Psychology, n ${ }^{\circ}$ 65, p. 178-182.
Arcimoles (D’), Charles-Henri; TrebucQ, Stéphane (2002) «Ressources humaines et création de valeur : Essai de modélisation et application à l'actionnariat salarié» dans F DupuichRabasse et al.; (sous la direction de), Gestion des compétences et knowledge management, Editions Liaisons, p. 23-35.

BASLE, Maurice (2009a) «Révision générale des politiques publiques et activités évaluatives en France : différences, complémentarités?», dans S. TROSA (sous la direction de), «Evaluer les politiques publiques pour améliorer l'action publique. Une perspective internationale, comité pour l'histoire économiques et financière de la France».

Bartoli, Anne; Chomienne, Hervé (2007). «Le développement du management dans les services publics : évolution ou révolution?», Les cahiers français, n³39, La Documentation française, juillet-août 2007, pp. 42-48 (réédité dans Informations Sociales «Le management du social» $\mathrm{n}^{\circ} 167-$ Sept.-Oct. 2011).

Bezes, Philippe; Demaziere, Didier (2011). Introduction de «New Public Management et professions dans l'Etat : au-delà des oppositions, quelles recompositions?» Sociologie du Travail, 53(3), p. 293-305.

Carassus, David; Favoreu, Christophe; Gardey Damien; Marin Pierre (2012). «La caractérisation et le management des déviances organisationnelles liées à la mise en œuvre d'une démarche de performance publique : application au contexte public local français ", Revue Management International, vol. 16, $\mathrm{n}^{\circ} 3$, p. 102-117.

Chappoz, Yves; Pupion Pierre-Charles (2013). «La quête de la performance» Gestion et Management Public Vol 1/10 3 .

Charreaux, Gérard (1998). «La mesure de la performance des entreprises» Banque \& Marchés, mai-juin n³4 p. 46-51.

Corbel, Pascal (2015). «Valorisation de la recherche publique : la difficile question des indicateurs de mesure de la performance», Revue de Management et de Stratégie, vol.1, n 1, p.1-9.

Cosnefroy, Laurent (2004). «Apprendre, faire mieux que les autres, éviter l'échec : l'influence de l'orientation des buts sur les apprentissages scolaires». Revue française de pédagogie, $\mathrm{n}^{\circ} 147$ avril-mai p.107-128.

Cusset, Pierre-Yves (2011). "Que disent les recherches sur l'effet enseignant?» note d'analyse $\mathrm{n}^{\circ} 232$ du centre d'analyse stratégique.

Decotiss Thomas; Summer Timothy (1987). «A path analysis of a model of the antecedents and consequences of organizational commitment», Human Relations, Vol. 40, N 7, p. 445-470.

Delery John.E; Doty D.Harold (1996). « Modes of theorizing in strategic human resource management: Tests of universalistic, contingency, and configurational perspectives». Academy of Management Journal, n’39 p.802-805

Emery Yves; Giauque David (2005). Paradoxes de la gestion publique, Editions L'Harmattan, Paris.

Emery Yves; Wyser Carole; Martin Noémi; Sanchez Joëlle (2008) «La perception de la performance par les agents publics suisses dans un environnement en rapide évolution ", Revue Internationale des Sciences Administratives 2008/2, p. 327-344.

Fournier, Sandrine (2015). «Pratiques managériales dans les EPLE et implication des enseignants » Revue Gestion et Management Public vol.3 nº 4.p..27-48. 
FOURNIER, Sandrine (2016). «Le stress et la performance au travail: le cas des enseignants " Revue Vie et sciences de l'entreprise 2016/2 n 202 p. $10-26$.

Gauthier Clermont; Bissonnette Steve; Richard Mario (2013). Enseignement Explicite et réussite des élèves. Gestion des apprentissages Montréal, Canada : ERPI.

Gordon, Michaël E; Philpot, John W; Burt, Robert E; Thompson, Cynthia A; SPILLER, William E (1980). Commitment to the union: Development of a measure and an examination of its correlates. Journal of Applied Psychology, Vol 65(4) p. 479-499.

Guenoun, Marcel (2009). «Le management de la performance publique locale : Etude de l'utilisation des outils de gestion dans deux organisations intercommunales", Thèse en Sciences de gestion, Université Paul Cézanne, Aix-Marseille III.

Greenhaus, Jeffrey H (1971). «An investigation of the role of career salience in vocational behavior" Journal of Vocational Behaviour, 1, p. 209-216.

Huselid, Marc.A. (1995). «The impact of Human Resource Management Practices on Turnover, Productivity, and Corporate Financial Performance». Academy of Management journal, vol. $38, n^{\circ} 3$, p. 635-672.

JoRgEnsen, T.Beck. (1999). «The public Sector in an in-between Time: Searching for new Public Values », Public Administration 77(3), p.565-84.

Lodhal Thomas; M., et KejNer Mathilde (1965). «The definition and measurement of job involvement». Journal of Applied Psychology, 49(1), p. 24-33.

Mac Duffie John Paul (1995). «Human resource bundles and manufacturing performance organization ». Industrial \& Labor Relations Review, 48(2), 197.

Manville Caroline (2014). «Parce que je le vaux bien», les relations entre caractéristiques du travail, estime de soi et engagement affectif envers l'organisation revue de gestion des ressources humaines $\mathrm{n}^{\circ} 92$ avril-mai-juin 2014.

Maroy Christian (2006). Ecole, régulation et marché : une comparaison de six espaces scolaires locaux en Europe, Presses universitaires de France, coll. Education et sociétés, 390 p.

Mazouz Bachir; Garzon César; Picard Pascal (2012). «Les déviances dans les organisations publiques en quête de performance : vers une gestion prophylactique des risques de déviance», Management international, 16, 3, p. 92-100.

Meyer John P; Allen Natalie (1984). Testing the "Side-Bet Theory" of Organizational Commitment: Some Methodological Considerations. Journal of Applied Psychology, 69(3), p. 372-378.

Meyer John P; Allen Natalie (1991). «A three-component conceptualization of organizal commitment ", Human Resource Management Review, vol. 1, nº 1, p. 61-89.

Meyer John P; Smith Catherine.A (2000). HRM practices and organizational commitment: Test of a mediation model. Canadian Journal of Administrative Sciences, Vol. 17, p. 319-331.

Meyer John P; Becker Thomas.E; Vandenberghe Christian (2004). «Employee Commitment and Motivation: a Conceptuual Analysis and Integrative Model », Journal of Applied Psychology, vol. $89, \mathrm{n}^{\circ} 6$, p. 991-1007.
Motowildo Stephan J. (2003). «Job performance», dans Handbook of Pyschology: Industrial and Organizational Psychology, vol. 12 , p. 39-53.

Mowday Richard.T; Porter Lyman.W; Steers Richard.M (1982). Employee-Organisation linkages: the psychology of commitment, absenteeism and turnover, New york, Academic Press

Neveu, J-P. (1996). La démission du cadre d'entreprise - étude sur l'intention de départ volontaire, Economica., $181 \mathrm{p}$.

Pulakos Elaine D; Arad, Sharon; Donovan, Michelle.A; Plamondon Kevin.E. (2000). "Adaptability in the workplace: development of a taxonomy of adaptive performance». Journal of Applied Psychology, vol. 85, issue 4, p. 612-624

Pfeffer Jeffrey; Veiga John F (1999). «Putting People First for Organizational Success ", The Academy of Management Executive, vol. 13, n 2, p.37-48.

Ferris Gerald R; Arthus Michelle M; Berkson Howard M; Kaplan David M; Harell-Cook Gloria. et Frink Dwight D (1999). «Toward a social context Theory of the Human Resource Management Organization effectiveness relationship " Human Resource Management Review, Fall 98, Vol. 8, Issue 3, p. 235-266

OCDE (2014). Regards sur l'éducation: Les indicateurs de l'OCDE, Éditions OCDE.

OCDE (2015). La rémunération liée aux performances dans l'administration, Éditions OCDE.

OCDE (2016). PISA les résultats à la loupe, Éditions OCDE.

OCDE (2017). Panorama des administrations publiques 2017, Éditions OCDE, Paris. Pirola-Merlo, Andrew; Hartel Charmine; Mann, Léon; Hirst Giles (2002). «How leaders influence the impact of affective events on team climate and performance in R\&D teams ", Leadership Quartely, Vol. 13, n5, 2002, p 561-581.

Pollitt, Christopher (2000). «Reinvention and the Rest: Reform Strategies in the OECD World» dans Y.Emery «L'administration dans tous ses états. Réalisations et conséquences », p.217-236. Lausanne, Presses Polytechniques et Universitaires Romandes.

Pollitt, Christopher; Bouckaert, Geert (2004). Public Management Reform: a comparative analysis. Oxford, Oxford University Press, $362 \mathrm{p}$.

Rhoades, Linda; Eisenberger, Robert; Armeli, Stephen (2001). "Affective commitment to the organization: the contribution of perceived organizational support, " Journal of Applied psychology, Vol. 86(5), p.825-836.

Rouban, Luc (2008) «Reform without doctrine: public management in France», International Journal of Public Sector Management, Vol. 21 Issue 2, p. 133-149.

Thevenet Maurice (1992). Impliquer les personnes dans l'entreprise, Paris, Editions Liaisons, 205 p.

VRoom Victor (1964). «Work and Motivation», New York : John Wiley \& Sons

Walumbwa Fred O; Avolio Bruce; Zhu Weichun (2008). «How transformational leadership weaves its influence on individual job performance: the role of identification and efficacy beliefs » Management Department Faculty Publications. 6.

Wood, Stephen; Albanese Maria Teresa. (1995). «Can we speak of Human Resource Management on the shop Floor?» Journal of management Studies, vol. 32, issue 2 p. 215-247. 


\section{ANNEXE 1}

Résultats de l'Académie de Bordeaux-Poitiers en 2016

\begin{tabular}{|l|c|c|c|c|c|c|}
\hline Types Etablissements & Nombre & Performant & Accompagnateur & Sélectif & $\begin{array}{c}\text { Neutre } \\
\text { En-deçà des } \\
\text { attentes }\end{array}$ \\
\hline Lycée Généraux et Techniques & 158 & $\mathbf{1 0}$ & 7 & 17 & 72 & 52 \\
\hline Lycées Professionnels & 154 & 19 & 7 & 21 & 55 & 52 \\
\hline Pourcentage (\%) & 100 & $\mathbf{9 , 3 0}$ & 4,49 & 12,18 & 40,70 & 33,33 \\
\hline TOTAUX & 312 & $\mathbf{2 9}$ & 14 & 38 & 127 & 104 \\
\hline
\end{tabular}

\section{ANNEXE 2}

\section{Unités de sens et extraits des items des échelles retenues}

\begin{tabular}{|c|c|}
\hline Echelles retenues & Items \\
\hline $\begin{array}{l}\text { Implication psychologique: } \\
\text { Lodalh 1965, Lodalh et Kejner } \\
1965, \text { Kanungo } 1982 \text { puis Neveu } 1996\end{array}$ & $\begin{array}{l}6 \text { items sont conservés concernant l'identification psychologique des enseignants: } \\
\text { l'implication psychologique, } 4 \text { items sont conservés sur la place émotionnelle du travail } \\
\text { dans la vie des enseignants concernant l'attachement au travail. }\end{array}$ \\
\hline$\frac{\text { Implication affective, normative et }}{\text { calculée d'Allen et Meyer (1990) }}$ & $\begin{array}{l}5 \text { items sont conservés pour l'implication affective au métier, } 3 \text { items sont conservés } \\
\text { pour l'implication affective à l'organisation, } 2 \text { items sont conservés pour l'implication } \\
\text { normative, } 2 \text { items sont conservés pour l'implication calculée sur l'attachement à } \\
\text { l'établissement, } 2 \text { items sont conservés pour l'implication calculée sur l'attachement à } \\
\text { la profession d'enseignant. }\end{array}$ \\
\hline Implication professionnelle & $\begin{array}{l}3 \text { items sont conservés pour la satisfaction quant aux conditions liées au pilotage, } 2 \text { items } \\
\text { sont conservés pour temps consacré au travail, } 2 \text { items sont conservés pour suivi des } \\
\text { élèves, } 2 \text { items sont conservés pour le temps de travail supplémentaire consacré aux } \\
\text { élèves dans l'établissement. }\end{array}$ \\
\hline $\begin{array}{l}\text { Performance collective Borman, Buck } \\
\text { and al. (2001),Coleman, Borman (2000) }\end{array}$ & $\begin{array}{l}4 \text { items sont conservés pour l'ambiance au travail (deux concernant l'ambiance } \\
\text { collective, deux concernant l'effet organisation de l'établissement sur le collectif). }\end{array}$ \\
\hline$\frac{\text { Performance individuelle }}{\text { Williams et Anderson (1991) }}$ & $\begin{array}{l}4 \text { items sont conservés pour le suivi des résultats par les enseignants } 3 \text { items sont } \\
\text { conservés pour la performance des enseignants et répercussions sur les élèves. }\end{array}$ \\
\hline$\frac{\text { Performance et stress }}{\text { Williams et Anderson (1991) }}$ & $\begin{array}{l}\text { - } 2 \text { items sont conservés pour les effets du rythme sur la performance,- } 3 \text { items sont } \\
\text { conservés pour les effets du stress sur la performance individuelle. }\end{array}$ \\
\hline
\end{tabular}

\section{ANNEXE 3}

\section{Modèle de recherche 1}

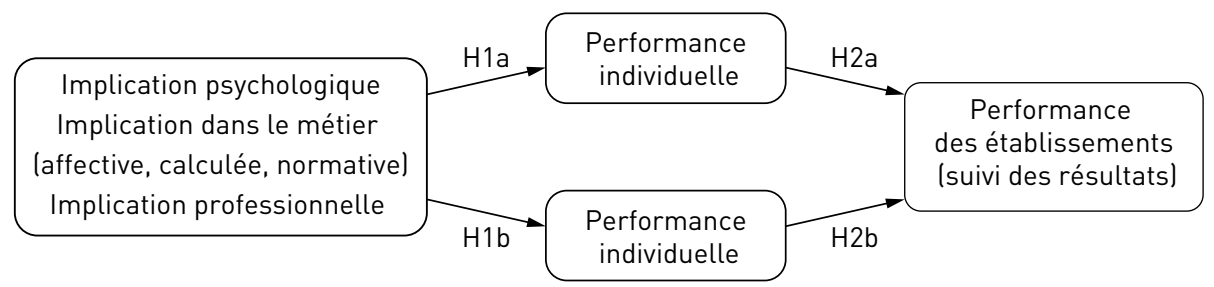




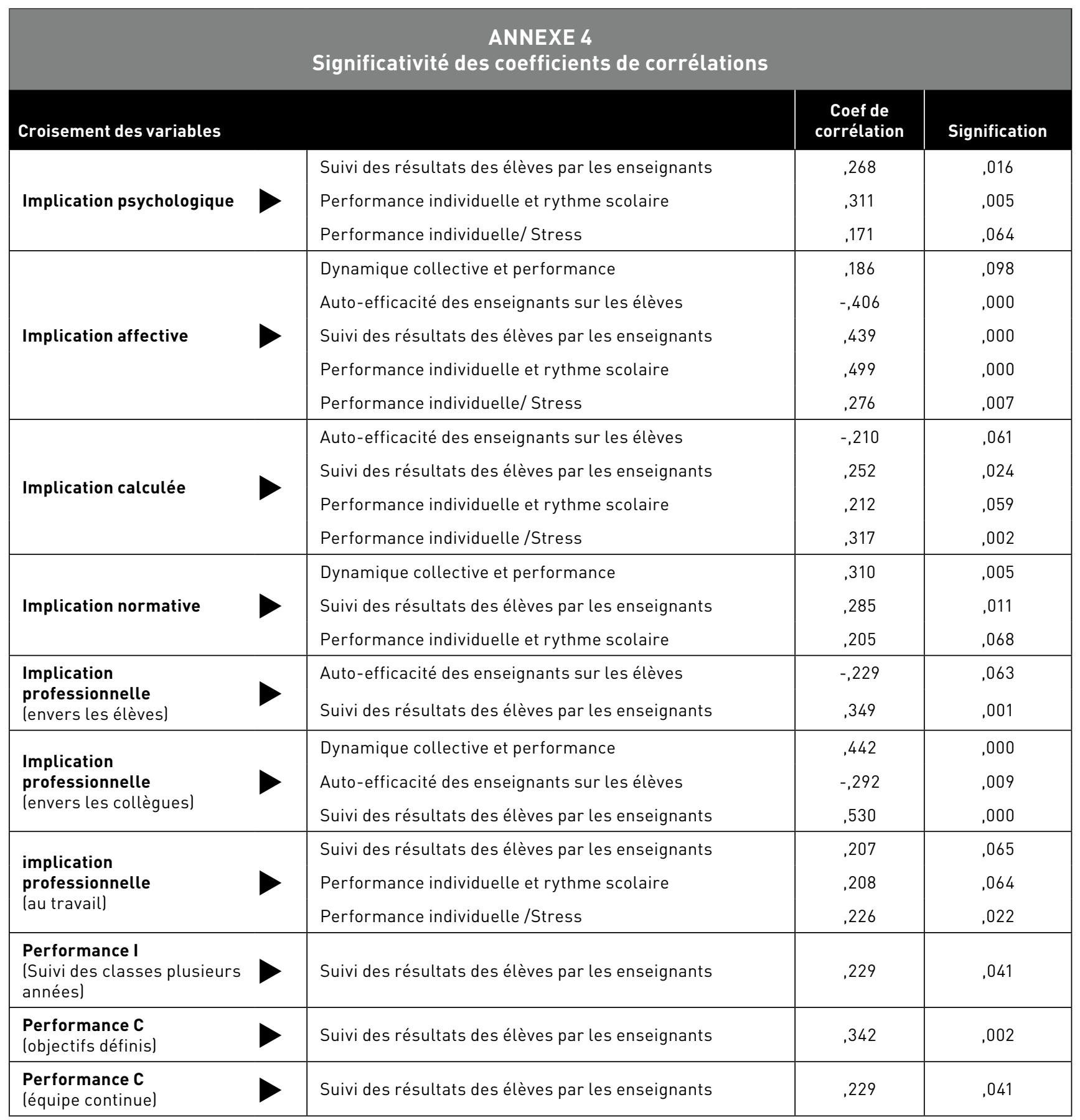

\section{ANNEXE 5}

Modèle de recherche à l'issue des résultats

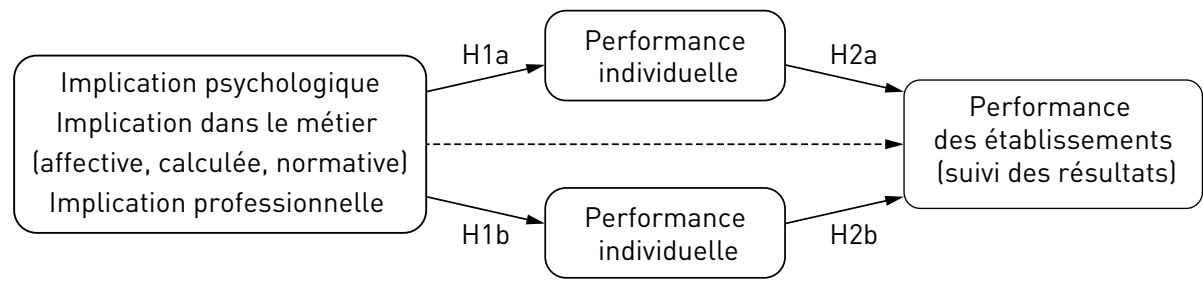

